\title{
LOS PLANES DE CIRCULACION COMO ACTUACIONES URBANAS A CORTO PLAZO
}

711.7

por

\section{Rafael Izquierdo}

Doctor Ingeniero de Caminos, Canales y Puertos Economista

Encargado de la Cátedra de aEconomía y Coordinación de Transportes» en la E. T. S. de Ingenieros de Caminos

\author{
José Luis Zubieta \\ Ingeniero de Caminos, Canales y Puertos \\ Economista \\ Profesor de aUrbanismo y Transporte Urbano \\ en la E. T. S. de Ingenieros de Caminos
}

SUMARIO: I. LOS COSTES DE LA CIRCULACION EN NUESTRAS CIUDADES.-II. ACTUACIONES POSIBLES.-III. LOS PLANES DE CIRCULACION.-IV. LOS ELEMENTOS DE LOS PLANES DE CIRCULACION: 1. ANálisis de la Situación actual. 2. Datos de base PARA los CÁlCuLOS. 3. ESTUdio DE LAS disTINTAS ACTUACIONES.-V. LA PLANIFICACION DEL ESTACIONAMIENTO: 1. IDEAS GENERALES DE LOS PROGRaMas De estacionamiento. 2. Datos NeCESARIOS PaRa EL DISEÑO DEL MODELO Y SU APLICACIÓN. 3. FUNCIONAMIENTO Y UTILIZACIÓN DEL PROGRAMA.-VI. IDEAS CONCLUSION EN FORMA DE RESU. MEN.

\section{LOS COSTES DE LA CIRCULACION EN NUESTRAS CIUDADES}

El habitante de nuestras ciudades se ve sometido a ritmo creciente a una amplia serie de perjuicios, desventajas o efectos no deseados, que en términos económicos pueden identificarse con los conceptos externalidades, costes sociales o deseconomías de escala, según los casos. 
Estos «males» públicos son consecuencia de un conjunto de concausas entre las que destacan el aumento del grado de urbanización, la concentración de los sectores económicos secundario y terciario en las ciudades y el avance de la motorización al elevarse el nivel de renta de la población.

En concreto, el aumento del grado de motorización da lugar a uno de los grandes problemas ciudadanos: la circulación urbana, causante de muchos de esos efectos no deseados. La presencia en la ciudad actual de un gran número de servicios, de empleos, de lugares de distracción, ofrece al ciudadano las opciones más variadas; los desplazamientos que se realizan en consecuencia, ocasionan, si se utilizan los vehículos privados, un cierto número de desventajas: ruido, contaminación, inseguridad para peatones, pérdidas de tiempo, etc.

En nuestro país el problema de tráfico urbano reviste quizá más virulencia que en otros países occidentales. Ello es debido al rápido crecimiento del número de automóviles en pocos años, a la inadecuación de los sistemas de transporte colectivo y a la propia estructura de nuestras ciudades, muchas de ellas con centros o zonas muy antiguos, con redes viarias no aptas para la circulación masiva. La política urbanística no ha contribuido precisamente a mejorar la situación.

La aparición de costes públicos, en concreto los debidos a la circulación urbana, es consecuencia inmediata de la existencia de una aglomeración, aunque el volumen de los mismos no sea proporcional al número de habitantes de la ciudad. Uno puede preguntarse a partir de qué número de habitantes, como término medio, las ciudades españolas comienzan a soportar unos costes debidos al tráfico urbano, que exijan adoptar ciertas soluciones y cuáles han de ser éstas. El tema, planteado en forma teórica por diversos investigadores de la economía urbana, enlaza directamente con otro más amplio: el tamaño óptimo de la ciudad. A su vez puede distinguirse entre el punto de vista público nacional, que considera a cada ciudad como integrante de la estructura del sistema de ciudades, y el punto de vista local al que vamos a referirnos. Para los ciudadanos, la ciudad habrá de crecer hasta que se maximice la renta disponible, diferencia entre el producto medio per capita y el coste medio per capita; desde el punto de vista de los entes públicos locales el tamaño de la ciudad debería aumentar hasta que 
la renta fuese máxima, es decir hasta que se igualasen los costes marginales y los productos marginales (1).

La partida de costes en la ciudad tiene varios componentes, pero podemos concretarlos en dos: dotaciones en infraestructuras y costes sociales por desventajas de aglomeración debidas en gran parte al tráfico urbano. Ambos componentes crecen en forma muy distinta con el número de habitantes de la ciudad, siendo mucho más rápida en el caso del segundo.

De los pocos estudios en este sentido aplicados a la realidad española puede fijarse la cifra $80.000-100.000$ habitantes como el tamaño de la ciudad a partir del cual la diferencia entre las economías y deseconomías externas marginales comienza a ser negativa, desde el punto de vista de la ciudad aislada y a corto plazo (2).

En consecuencia, y al margen de la política de crecimiento de las ciudades, podemos resumir que las ciudades españolas que superan los 80-100.000 habitantes empiezan a soportar unos costes excesivos, en gran parte debidos al tráfico urbano, que obliga a la adopción de medidas para tratar de reducirlos.

\section{ACTUACIONES POSIBLES}

Parece evidente la afirmación de que los inconvenientes de la situación actual exigen soluciones inmediatas si no se quiere que los costes sociales sigan aumentando, o en palabras más claras, que sea mayor la amenaza de asfixia por la circulación en muchas ciudades.

Lo que supone mayor dificultad es definir y jerarquizar el conjunto de actuaciones posibles, y aún más el intentar evaluar la rentabilidad de la aplicación de alguna de ellas.

A cualquier nivel, las actuaciones que afectan al tráfico urbano se concretan, como tantas otras, en decisiones políticas y vienen determinadas, en consecuencia, por unos parámetros socioeconómicos específicos. El transporte y la circulación urbanos, como una expresión más de las relaciones sociales, puede observarse con la óptica que en sociología o ecología humana representa la pugna funcionalismo-dialéctica.

(1) Una amplia discusión del tema del tamaño optimo de la ciudad puede verse en la bibliografía [1] y [2].

(2) Véase [1], págs. 26 y ss. 
Las decisiones políticas en los niveles más generales, que naturalmente trascienden el problema de la circulación urbana, descienden cada vez más condicionados a los niveles de actuación más particulares y a corto plazo. Los técnicos, en cuanto tales, han de contemplar la problemática con la suficiente objetividad como para adaptarse al tipo de solución que se le proponga y ofrecer la optimización técnica, al margen de su opinión política sobre la medida. Esto parece especialmente válido para las soluciones a corto plazo que se analizan en estas páginas y que son especialmente discutibles desde perspectivas más elevadas.

El antagonismo o conflicto transporte público-transporte privado es posiblemente el elemento primordial en la política del tráfico urbano y su planteamiento, en términos técnicos o económicos, el más difícil y polémico. No parece exagerado decir que cualquier medida que se decida en el ámbito del tráfico urbano, estará favoreciendo a uno de los dos sistemas $o$, al menos, a uno más que otro. Por eso será fácil identificar incompatibilidades a la hora de decidir actuaciones que tengan como objetivos mejorar las condiciones de circulación y al tiempo potenciar el transporte público.

El presente artículo no va a referirse a los planteamientos generales del problema del tráfico urbano como los de BUCHANAN, SMEEd o BEESLeY y KaIN (3) que proponen soluciones en términos sintéticos, como un conjunto de medidas posibles, económicas y urbanísticas. La intención es conseguir un cierto orden de estas medidas para desarrollar las de acción a corto plazo, que se concretan en los Planes de Circulación.

Como antecedente y punto de referencia por su exhaustividad, interesa exponer la clasificación de medidas propuesta por la Conferencia Europea de Ministros de Transporte (4).

\section{A) Medidas que no exigen inversiones importantes}

- Utilización de infraestructuras

Se agrupan bajo este epígrafe un conjunto de medidas que se enumeran a continuación.

La primera corresponde a una mejora de la distribución hora-

(3) Principalmente en los informes o artículos citados en la bibliografía [3], [4] y [5].

(4) Que se recoge con detallada exposición de estas medidas en [6]. 
ria de utilización de las vías actuando principalmente sobre los horarios de trabajo y comercio y, en general, por medio de lo que se denomina planificación del tiempo.

Las medidas de orden administrativo forman otro grupo, entre las que se encuentran la aplicación de las reglas de circulación, prohibición de estacionamiento, etc.

Las medidas con incidencia financiera incluyen tanto la tarificación por el uso de los transportes públicos, posible gratuidad, tarifas subvencionadas, etc., como la tarificación por el uso de las infraestructuras, tasas de estacionamiento o penetración en el centro, tarificación global, etc.

Por último, dentro de este grupo, hay que referirse a las medidas que sirven de orientación o fomento en la elección de un determinado medio de transporte, utilizando medios distintos de los administrativos o tarifarios, ya comentados. Esto puede conseguirse por vía fiscal o por una mejora de la oferta del transporte público.

\section{- Gestión de las empresas de transporte público}

Agrupa a otro conjunto de medidas entre las que destacan las siguientes:

Medidas de coordinación que pueden ser de tipo administrativo, geográfico o técnico además de las que afectan directamente a las autoridades coordinadoras.

En algunas ocasiones podrá procederse a una nueva repartición de servicios entre empresas, modificando sus redes o itinerarios y uniformando la gestión y la presentación de la oferta.

Otras medidas en el mismo sentido pueden ser la reducción del múmero de explotaciones y la estructuración óptima de las empresas.

\section{B) Medidas que exigen inversiones importantes}

Se refieren en este grupo el conjunto de medidas de mayor amplitud y a más largo plazo, relacionadas con la planificación regional y urbana, nuevas ciudades, nuevos sistemas de transporte colectivo, etc., en cuyo detalle no se va a entrar (5).

(5) La clasificación y análisis de estas medidas puede seguirse en [6], págs. 472 y 489 y ss. 
La clasificación a utilizar, en definitiva, para enmarcar los Planes de Circulación es mucho más sencilla:

- Medidas que exigen inversiones importantes.

- Medidas sobre las empresas de transporte urbano colectivo (técnicas y económicas).

- Medidas fiscales o de tarificación sobre el transporte privado.

- Medidas para la utilización óptima de las infraestructuras existentes.

\section{LOS PLANES DE CIRCULACION}

Al conjunto de medidas comprendidas en el apartado cuarto anterior, se llama «Plan de Circulación» el cual podría definirse como el conjunto de actuaciones técnicas y reglamentarias sobre el tráfico en la ciudad que se caracterizan por tener validez a corto plazo y no exigir unas inversiones elevadas.

Esta definición no ha de entenderse en sentido estricto, pues en algunos casos particulares los Planes de Circulación pueden afectar a medidas clasificadas en alguno de los otros tres grupos. Así pueden existir:

a) Medidas que exigen inversiones, cuando de los estudios necesarios para la elaboración del Plan de Circulación se deduzca la urgencia de alguna inversión concreta y aislada en infraestructura, como un aparcamiento subterráneo o un cruce a desnivel.

b) Medidas que afectan directamente al servicio del transporte colectivo, como pueden ser la creación de carriles-bus, la modificación de itinerarios o de ubicación de paradas.

c) Medidas económicas o de tarificación como, por ejemplo, modificación de las tarifas de aparcamientos o parkímetros.

Otra forma de precisar el concepto general de los Planes de Circulación es tratar de distinguir sus objetivos fundamentales. Tres son los niveles a considerar:

1. Mejorar las condiciones de circulación y la fluidez del tráfico, permitiendo unas velocidades más elevadas que acorten los tiempos de viaje.

2. Reducir los accidentes urbanos, en particular los que afec$\tan$ a los peatones. 
3. Mejorar, o al menos preservar, las condiciones del medio ambiente, reduciendo las desventajas generadas por el tráfico y creando zonas separadas de la circulación, como las vías reservadas a los peatones (zonas peatonales).

Los estudios que requiere la elaboración de un Plan de Circulación con todas las medidas, no ha sido emprendido todavía en las ciudades españolas, salvo en forma parcial. Los antecedentes programáticos de la Administración, tampoco son muy explícitos en este sentido. Así la monografía sobre Estructuras y Servicios Urbanos del III Plan de Desarrollo, constata la gravedad de la situación creada por un aumento de la demanda de transporte privado no seguido por el de la oferta de capacidad viaria (6), propugnando una política fundamentada en conseguir una coordinación de los transportes colectivos existentes y fomentar éstos frente al transporte privado moderando la demanda de éste último, por medio de actuaciones sobre las tarifas y subvenciones (7). En otros capítulos se hace referencia a las inversiones más o menos importantes en infraestructuras viarias (redes arteriales) y específicas de sistemas de transporte urbano colectivo, sin incluir una mención específica a las medidas más modestas, pero no menos importantes, que componen un Plan de Circulación.

El balance de las actuaciones en este sentido en nuestras ciudades durante el período de vigencia del III Plan es bastante pobre. Si exceptuamos las inversiones en metropolitanos y el planeamiento de nuevas redes, difícil es encontrar otros ejemplos de estudios o medidas prácticas sobre la circulación.

Diversos países europeos disponen de legislación sobre este tipo de actuaciones, pero quizá sea Francia el que aporte una instrumentación técnica y administrativa más avanzada. Las ciudades francesas presentan problemas que con facilidad pueden compararse con los de las españolas y ello por motivos históricos, culturales, etc., a pesar del mayor grado de desarrollo y por tanto de motorización.

El programa de los Planes de Circulación franceses (Plans de Circulation) fue puesto en marcha por una circular interministerial (Equipo e Interior) de abril de 1971. Durante 1972 y 1973 se realiza

(6) Páginas 314 y ss., nota bibliográfica [7].

(7) Ibidem, págs. 316, 317. 
una serie de estudios pilotos elaborándose las normas generales para la redacción de los Planes de Circulación y la solicitud de los créditos correspondientes a su ejecución. Por último, ya en 1975 se han modificado algunos aspectos institucionales, englobándose los Planes de Circulación con los Programas de Infraestructuras (Programme des Infrastructures) en una nueva orientación bajo el nombre de Dossier d'agglomeration. La unión de los dos conjuntos de actuaciones ha obligado a la consecución oficial de créditos durante el VII Plan Francés 1976-80.

Sin detenerse más en el montaje institucional de los Planes de Circulación franceses, hay que destacar que las ciudades que deciden hacer un Plan de Circulación reciben una doble participación del Estado: parte del Ministerio del Equipo y parte del Ministerio del Interior (8).

Por último, y antes de pasar a exponer los distintos elementos de los Planes de Circulación, interesa repetir una idea, que, aunque implícita en otros lugares de estas notas, parece de especial importancia. Aunque los capítulos siguientes sólo hacen referencia a los Planes de Circulación sin relación con las otras medidas citadas anteriormente, no parece que pueda preconizarse como medida única y aislada a tomar en ninguna ciudad.

Con excepción de ciudades muy pequeñas, donde el transporte público tenga muy poca importancia, puede señalarse como norma general la utilidad de la realización de un estudio de los transportes coelctivos urbanos, paralelamente al Plan de Circulación. Podría incluso pensarse en una inclusión de este estudio en el Plan de Circulación, pero metodológicamente es más riguroso distinguir entre ambos trabajos, pues los objetivos del primero pueden distanciarse de los del segundo. Así un estudio de los transportes colectivos, puede buscar no solamente la optimización del servicio desde el punto de vista de la adecuación oferta-demanda, sino otros fines diferentes como el análisis de la tarificación, concesiones de líneas, equilibrios económico-financieros de las empresas explotadoras, etcétera.

Al defender la realización paralela de un Plan de Circulación, con el contenido que más adelante se detalla, y un estudio de los transportes urbanos colectivos y la toma de decisiones como con-

(8) Una información más extensa sobre los distintos aspectos técnicos o institucionales de los Planes de Circulación franceses, puede conseguirse en las publicaciones oficiales del Ministerio del Equipo. Véase [8]. 
secuencia de los resultados de ambos, se anulan algunas de las oposiciones o dudas teóricas que pueden plantearse ante los Planes de Circulación como medida independiente.

\section{LOS ELEMENTOS DE LOS PLANES DE CIRCULACION}

El esquema de los distintos pasos y metodología de elaboración de un Plan de Circulación depende de la ciudad concreta para la que se realiza y su problemática. Además, no parece válido el fijarse demasiado en los programas generales preparados para los planes franceses, que se han citado, pues la complejidad administrativa de su aprobación exige distintas etapas en el estudio que progresá a medida que se aprueban los créditos.

La no institucionalización de los Planes de Circulación en Es. paña, permite desarrollar un esquema general de los mismos, in. dependiente de los condicionamientos legales o administrativos que, en su dia, pudieran afectarles.

Pueden fijarse también los límites de población de las ciudades a las que se puede aplicar este esquema general. El mínimo de 80-100.000 habitantes ha sido comentado anteriormente. Un máximo de 600-800.000 parece también razonable; a partir de este tamaño la complejidad del funcionamiento de la ciudad puede invalidar alguno de los planteamientos parciales del Plan de Circulación, exigiendo otros más completos que integren la planificación del transporte colectivo, la inversión en infraestructura de red arterial, etc.

Con estas premisas se puede abordar la exposición de los pạsos principales de un Plan de Circulación.

\section{Análisis de la Situación actual}

Este análisis ha de fundamentarse en el conjunto de documentación, datos o estudios previos que existan sobre la ciudad. Por esto su amplitud dependerá de la documentación existente. En cualquier caso habrá que conseguir un mínimo de información que se detalla a continuación.

El diagnóstico de la situación de la circulación en la hora punta puede conseguirse de manera eficiente por medio de una serie de 
fotos aéreas. Este método, aunque costoso, da buenos resultados en la mayoría de los casos. Con la fotografía aérea se detectan tanto los tramos saturados como los cruces críticos o muy obstruidos en un momento determinado, contribuyendo a apreciar o decidir un orden de prioridad en los sectores sobre los que se ha de actuar. La obtención de fotos consecutivas separadas unos segundos, permite calcular las velocidades de los vehículos en cada tramo y los tiempos pasados en los cruces.

Es claro que toda la información que se consiga por medio de esta técnica puede también obtenerse por otros medios, pero el alto costo de la realización de un vuelo puede merecer la pena si se tiene en cuenta el elevado número de personas necesarias para el trabajo terrestre. Aún así será necesario en algunos casos la realización de algún trabajo para completar o suplir los resultados de los vuelos.

Las estadísticas de accidentes urbanos existentes, contribuyen a conocer el grado de seguridad de cada zona, vía, etc. Según sea la calidad de esta información podrán elaborarse índices de peligrosidad de distintos tipos, referidos a la zona, tipo de vehículo o peatones, llegándose incluso a realizar cálculos de los costes de estos accidentes y evaluación de los evitados por una posible solución o mejora.

Más difícil será disponer en muchas ciudades de estadísticas de ruidos o contaminación que sean operativos, pero las existentes contribuirán al diagnóstico de la situación.

Por último será necesario estudiar una serie de datos de tipo histórico, cultural o artístico de la ciudad que enmarcarán una visiọ́n general del problema.

Del estudio de toda esta información se deducirán los primeros resultados sobre la situación actual. Además de una descripción general de la situación de la circulación, podrán elaborarse gráficos y tablas con índices de saturación, velocidades de circulación, etc., junto con los datos de seguridad, ruido y contaminación existentes.

Otro resultado muy importante de esta primera parte del estudio será la definición de zona centro de la ciudad en la cual los problemas de circulación presentan características especialmente agudas y a cuyos límites habrán de referirse ciertos datos del cálculo, encuestas O-D, etc. 


\section{Datos de base para los cálculos}

El diagnóstico realizado permitirá ya definir unas líneas de actuación, pero será necesario todavía conseguir un conjunto de datos base para los cálculos.

El planteamiento del estacionamiento, punto clave de los Planes de Circulación, como se verá, exige una información muy amplia.

En primer lugar hay que conocer la oferta de plazas de estacionamiento referidas a las distintas zonas en que se divida el centro, ya definido. Estas zonas habrán de coincidir, en lo posible, con las divisiones municipales de barrios y distritos, para que los datos obtenidos sean territorialmente homogéneos con el resto de información.

No es este el lugar para la explicación de los procedimientos a seguir para inventariar las plazas de estacionamiento, pues se encuentran en cualquier manual de ingeniería de tráfico, pero sí hay que destacar la importancia de distinguir no solamente las plazas en bordillo, sino las existentes en inmuebles de uso público o privado, garajes, así como las plazas en zona azul, con parkímetros o estacionamientos públicos, así como las tarifas de utilización en su caso.

Los modelos de estacionamiento que se explicarán más adelante, exigen para su identificación un conjunto de datos socioeconómicos de las distintas zonas de la ciudad que definan la demanda de viajes en automóvil con destino en cada una. La disponibilidad de unos u otros de estos datos y su calidad decidirá la elección del modelo de demanda más apropiado. Los más complejos utilizan diversos índices calculados a partir de los datos siguientes para cada zona: población, población activa, puestos de empleo secundario, puestos de empleo terciario, grado de motorización, etc. En nuestras ciudades no es corriente disponer de gran cantidad de valores de este tipo, por lo que los modelos de demanda han de adaptarse a los existentes.

Esto no es obstáculo para que en algunos casos sea preferible realizar ciertos estudios anteriores al Plan de Circulación, con el fin de conocer algunos de estos datos socioeconómicos que aseguren el éxito en la utilización de los modelos.

En este sentido merece la pena recalcar la múltiple utilidad de los estudios de este tipo cuyos resultados podrán ser interesantí- 
simos a la hora de abordar otros trabajos como pueden ser el planeamiento de la red arterial de la ciudad o de los sistemas de transporte colectivo.

Por último será necesario disponer de los resultados de una encuesta de orígenes y destinos y de otras encuestas y conteos parciales, según el tipo de modelo de estacionamiento utilizado, para el ajuste de los mismos. En algunos casos podrá disponerse de encuestas y conteos realizados en la ciudad con otros fines, como pueden ser los estudios de tráfico de las redes arteriales: en estos casos puede analizarse la posible utilización de estos resultados si se ajustan a las necesidades del trabajo.

La encuesta orígenes y destinos citada no ha de ser necesariamente complicada. Su utilización para el estudio de las vías unidireccionales sólo obliga a la zonificación del centro y periferia de la ciudad en un número pequeño de áreas (8-12) realizando la encuesta del tipo de cordón en el límite de la zona centro en el período punta del día. La matriz o matrices O-D así obtenidas suelen ser suficientes para diseñar un grafo de flujos en la hora de máxima punta. Otros datos como el conteo de peatones, inventario de comercios, etc., dependerán de cada estudio concreto.

\section{EstUdio DE LAS DISTINTAS ACTUACIONES}

Habiendo realizado un diagnóstico de la situación actual y disponiendo de los datos mínimos enumerados puede abordarse el estudio de las diferentes actuaciones que componen el Plan de Circulación.

A continuación se expone una clasificación de estas actuaciones, siguiendo un orden que puede considerarse tanto jerárquico, como de redacción del estudio.

A) Rupturas de carga.

A-1) Estacionamiento de los vehículos privados.

A-2) Delimitación de zonas peatonales.

A-3) Delimitación de zonas de carga y descarga.

A-4) Modificación de paradas de transporte colectivo.

B) Circulación.

B-1) Establecimiento de sentidos únicos y modificación de cruces y giros. 
B-2) Regulación por semáforos.

B-3) Señalización horizontal y vertical.

C) Medidas de seguridad.

\section{A-1) Estacionamiento de los vehículos privados}

La delimitación precisa de las zonas de estacionamiento y sus distintos usos, es uno de los fines esenciales de un Plan de Circulación. Esta medida, aunque haya de buscarse el equilibrio estacionamiento-circulación, puede considerarse básica en el Plan pues condiciona todas las demás.

Por otra parte es la actuación de los Planes de Circulación que mejor admite la utilización de programas de ordenador para su tratamiento, lo que permite obtener, al margen de los previos juicios de valor o hipótesis, los resultados más rigurosos.

Dada esta importancia, se deja para el apartado siguiente una exposición más detenida del planeamiento del estacionamiento.

\section{A-2) Delimitación de zonas peatonales}

Su estudio se basará en los conteos de peatones en las horas punta comerciales y densidad de comercios y se tendrá en cuenta la facilidad de acceso de las mercancías a los comercios de la zona.

La adecuada pavimentación de las vías reservadas a los peatones, colocación de bancos, maceteros, etc., permitirán conseguir el cuadro adecuado.

\section{A-3) Delimitación de zonas de carga y descarga}

Evidentemente relacionado con el planeamiento del estacionamiento de los vehículos privados, exige estudiar no sólo las zonas reservadas sino los horarios de las mismas.

En los casos de ciudades con ordenanzas municipales sobre carga y descarga, habrá que adaptarse a las mismas, aunque puede ser trabajo anejo al Plan de Circulación la elaboración de las normas básicas para la redacción de unas nuevas ordenanzas o modificación de las existentes. 


\section{A-4) Modificación de paradas de transporte colectivo}

Consecuencia inmediata de las medidas anteriores, puede aprovecharse para la mejora de los refugios e instalaciones de las paradas. En cualquier caso habrá de tener en cuenta otro punto de vista en la ubicación de las paradas de autobuses: la optimización de las distancias entre las mismas que puede calcularse en función de las velocidades y aceleraciones de los vehículos recorridos a pie de los viajeros.

\section{B-1) Establecimiento de sentidos únicos y modificación de cruces $y$ giros}

Los flujos de deseo de circulación, puestos de manifiesto por las matrices O-D, resultado de las encuestas correspondientes, son suficientes para estudiar distintas soluciones en el establecimiento de sentidos únicos. El examen comparativo de las variantes y definición de la mejor obligará a las modificaciones oportunas de cruces y giros.

En este grupo de medidas hemos de incluir también la implantación de carriles-bus, en calles de doble sentido o sentido único $y$ en este último caso a favor o en contra del resto del tráfico.

\section{B-2 y B-3) Regulación por semáforos y señalización}

Consecuencia también de las medidas anteriores. La regulación semafórica dispone de técnicas apropiadas de coordinación y cálculo de ciclos que se recogen en los manuales.

Tampoco la señalización horizontal o vertical precisa explicación especial.

\section{C) Medidas de seguridad}

Los Planes de Circulación más detallados han de contemplar los aspectos de seguridad, sobre todo del peatón. Algunas de las medidas anteriores contribuyen a mejorar la seguridad, pero pueden recordarse otras actuaciones posibles como mejora del alumbrado, elementos separadores vehículos-peatones, mejoras del pavimento, etcétera. 
REVL-1976, núm. 190. IZQUIERDO DE BARTOLOME, RAFAEL. LOS PLANES DE CIRCULACION C...

Los planes de circulacion como actuaciones uRbanas a CORTo plazo

363

\section{LA PLANIFICACION DEL ESTACIONAMIENTO}

Se ha hecho referencia anteriormente al papel de la planificación del estacionamiento en los Planes de Circulación y los motivos que justifican una referencia más detallada a esta parte.

Los estudios clásicos de estacionamiento, realizados en algunas de nuestras ciudades, presentan metodologías muy similares. Parten de un inventario de plazas en la zona definida como centro y una medición de la demanda real por medio de un aforo de cordón en los límites de la zona centro. La curva de demanda acumulada en el día, define la hora punta de estacionamiento a la que se refieren todas las soluciones que a continuación se analicen. Generalmente en estos estudios no se ha calculado una demanda potencial, función de los usos del suelo, pues en la hora punta este valor era superior a la capacidad de las vías de acceso y no podía ser nunca satisfecha.

Ultimamente se están poniendo a punto programas de ordenador que permiten alcanzar mejores resultados en el planeamiento del estacionamiento en el centro de las ciudades al optimizar las soluciones que se propongan y servir incluso de instrumentos de evaluación. El Ministerio del Equipo francés ha puesto a punto programas de este tipo para los Planes de Circulación (9). No se puede aspirar por el momento a utilizar estos mismos programas en nuestras ciudades, por falta de datos estadísticos suficientes sobre las características socioeconómicas de las distintas áreas del centro, pero si se pueden diseñar otros similares adaptados a los datos disponibles en las ciudades españolas.

\section{IDEAS GENERALES DE LOS PROGRAMAS DE ESTACIONAMIENTO}

Dos características básicas definen a este tipo de modelos. En primer lugar que se refieren, en todos sus cálculos, a la hora de máxima punta del día en el centro. Segunda, que solamente son operativos si la oferta total de plazas de estacionamiento es superior a la demanda. Respecto a esta última condición se supone además que la capacidad de las vías de acceso admite la demanda total prevista. Además, para calcular la oferta se tiene en cuenta

(9) Estos programas han sido publicados con amplias explicaciones sobre su alcance y operatividad. Véase [9]. 
no sólo las plazas de estacionamiento permitidas de todo tipo, sino también todo lugar apto para aparcar, aunque esté prohibido.

Los programas de estacionamiento permiten representar la ocupación de las plazas de estacionamiento en la hora punta, poniendo en concurrencia los datos de oferta y demanda de aparcamiento de cada una de las zonas en que se divide el centro.

El estudio se realiza en dos etapas. En la primera se representa la situación actual, ajustándose los modelos. En la segunda se utilizan los modelos así ajustados para probar distintas soluciones previstas con situaciones diferentes de la oferta de plazas.

\section{Datos NECESARIOS PARA EL DISEÑo DEL MODELO Y SU APLICACIÓN}

\section{- Oferta de plazas}

Calculada para cada zona a partir del inventario de plazas. Se ha de subdividir según todos los tipos distintos de plazas que se consideren, aparcamiento libre, aparcamiento prohibido, zona azul, parkímetro, garaje, etc.

- Demanda de plazas

La demanda de plazas en cada zona se entiende como una función del uso del suelo en la misma. Su cálculo ha de realizarse por un conjunto de modelos que pueden considerarse como un subprograma del programa de estacionamiento.

Si bien conceptual y teóricamente esto no presenta dificultades especiales, en la práctica resulta la parte más delicada, pues depende en cada caso de los datos disponibles (10).

Independientemente de la forma concreta de estos modelos, la demanda de cada zona se divide en tres sumandos: demanda residente, trabajo o larga duración y demanda de corta duración. Este mismo orden se sigue en la asignación de la demanda a la oferta, como se verá.

\section{- Distancia entre zonas}

La distancia entre las zonas es calculada por el ordenador a partir de las coordenadas del centro de gravedad de cada una, dato

(10) Los modelos concretos utilizados y ajustados en cl programa francés pueden verse en la publicación citada [9]. 
que se le aporta. También puede calcular una distancia interna en función de la superficie de la zona y que representa la distancia media de recorrido a pie de un automovilista, aunque haya conseguido estacionar en la zona a la que se dirigía.

\section{- Costes}

Varios valores de costes hay que introducir en el programa, alguno de los cuales exige cálculos previos.

En primer lugar se estima o calcula el valor del tiempo de desplazamiento a pie.

Por otra parte se introduce el precio de aparcamiento en plazas de pago en cada zona. Para las plazas de estacionamiento prohibido se ha de calcular en función del riesgo o probabilidad de multa y cuantía de la misma.

Diversos perfeccionamientos y mejoras permiten estos cálculos previos, como el estudio de los tiempos del parkímetro no consumidos $\mathrm{y}$, por tanto, válidos para otro usuario, etc.

\section{FunCIONAMIENTo Y UTILIZACIÓN DEL PROGRAMA}

El funcionamiento del programa se basa en una asignación de la demanda de las zonas a la oferta, buscando siempre el coste mínimo del aparcamiento más la marcha a pie.

Como la demanda de cada zona viene dividida en tres sumandos, se comienza asignando la demanda residente, primer sumando, empezando por las zonas más céntricas y siguiendo hacia el exterior. Si existe oferta de plazas gratuitas en la zona correspondiente se asigna la demanda a la misma y el coste que se considera es únicamente el del recorrido andando dentro de la zona (distancia interna). Si no existen plazas gratuitas en esa zona, se elige la opción más barata entre las restantes, bien una plaza de pago en la zona, bien una gratuita en una zona cercana. Cada vez que se realiza una asignación se contabiliza el coste de esa operación y la oferta residual en cada zona.

Una vez asignada toda la demanda residente se sigue con la demanda de viajes al trabajo y, por último, con la demanda de corta duración.

Como resultado final el programa puede dar los índices de ocu- 
pación de cada tipo de plazas en cada zona y los costes parciales y totales de la plaza más el desplazamiento andando.

No es necesario destacar la amplia utilidad de un programa de este tipo. Se puede analizar con él cualquier solución de aparcamiento que se prevea, sin más que variar adecuadamente la oferta de plazas de las zonas y los costes correspondientes. Así se puede analizar las consecuencias de creación de aparcamientos e inmuebles y sus distintas localizaciones, creación de zona azul, instalación de parkímetros y sus distintas tarifas, prohibición de estacionar en zonas, etc. Incluso puede estudiarse con estos programas las consecuencias de una mayor o menor vigilancia policial o efectividad de las denuncias, pues variará el riesgo de multa y por tanto el coste para los usuarios de las plazas de estacionamiento prohibido.

Como puede verse, estas últimas ideas señalan la posible, y por otra parte sencilla, utilización de estos programas en el análisis coste-beneficio de inversiones, aunque sean pequeñas, en este campo. Pero con ello se está ya fuera de los límites de un Plan de Circulación.

\section{IDEAS CONCLUSION EN FORMA DE RESUMEN}

Muchos de los conceptos expuestos en los apartados anteriores son evidentemente discutibles. La carga polémica que todo discurso acerca del tráfico urbano tiene, junto con la impopularidad que para unos u otros sectores representa la adopción de cualquier medida, convierte en opinable y, por tanto, modificable o reversible cualquier planteamiento sobre el tema.

Por todo ello se cierran estas notas con un conjunto de ideas resumen que repiten o intentan aclarar lo dicho anteriormente:

1. Es necesario reducir los costes creados por el tráfico urba. no. Las medidas posibles para ello son muy amplias.

2. Los Planes de Circulación no son las medidas más importantes. El primer lugar en esta jerarquía, al margen de medidas urbanísticas a largo plazo, parece reservado para la potenciación de los transportes colectivos.

3. En cambio, los Planes de Circulación reúnen las actuaciones más sencillas de llevar a cabo, al no exigir inversiones importantes. Por otra parte se trata de medidas reversibles, si las circunstancias cambian. 
4. Hay que destacar de nuevo lo que puede considerarse como finalidad fundamental de los Planes de Circulación: conseguir la utilización óptima de las infraestructuras existentes. Es claro que no tiene que coincidir «óptimo» con "máximo».

5. Alguna de las medidas incluidas en un Plan de Circulación puede parecer contradictoria con la que consideramos de mayor rango, potenciación del transporte público. Así la creación de plazas de aparcamiento en una zona puede fomentar el uso del vehículo privado con destino a la misma. En este sentido hay que analizar todas las medidas del Plan de Circulación de una forma global. Existirán algunas que analizadas parcialmente aparezcan fomentando el transporte privado, pero otras lo limitarán, como la creación de carriles-bus, limitaciones de aparcamiento, zona azul, creación de zonas peatonales, etc. Aun así puede suceder que el saldo final sea de una mayor capacidad de la red viaria, pero el resultado será válido si hemos reducido sustancialmente el conjunto de costes sociales del sistema.

6. Aunque el Plan de Circulación se base en el equilibrio estacionamiento-capacidad de las vías, la metodología del estudio parece que ha de fundamentarse en una planificación cuidadosa del estacionamiento, siendo las otras medidas consecuencia de la solución adoptada para el estacionamiento. Parece que la utilización de modelos programables para ordenador es más eficiente en el caso del planeamiento de los distintos tipos de aparcamiento que en el de análisis de las otras medidas de los Planes de Circulación. Los modelos clásicos de generación-atracción, distribución y asignación de viajes, válidos sin ninguna duda para el planeamiento de redes arteriales, pierden eficacia al aplicarse al conjunto de calles que forman la red viaria del centro de las ciudades.

7. No parece recomendable el abordar un Plan de Circulación en una ciudad de una forma aislada. Salvo en el caso de ciudades pequeñas, es complemento exigido al Plan de Circulación, el realizar un estudio de los transportes colectivos que como finalidad mínima busque la optimización del servicio. 


\section{BIBLIOGRAFIA}

1. J. R. LASUEN: aSobre desarrollo nacional y urbano», Cuadernos de Economía, II, núm. 3, Barcelona, 1974.

2. P.-H. DeRYCKE: La economia urbana, Madrid, Instituto de Estudios de Administración Local, 1971.

3. Ministry of TRANSPORT: Traffic in towns, London, H. M. S. O., 1963.

4. R. J. SMEED: aThe traffic problem in towns», Traffic Engineering and Control, VI, núm. 6, 1964.

5. M. E. BeEsley y J. F. KaIN: «Urban form, car ownership and public policy», Urban Studies, I, núm. 2, 1964.

6. Conférence Européenne des Ministres des Transports. Dix-septiéme rapport annuel et résolutions, 1970.

7. III Plan de Desarrollo. Estructuras y Servicios Urbanos, Madrid, 1972.

8. Les Plans de Circulation, París, Ministére de l'Equipement, 1974.

9. Etudes de stationnement, París, Ministére de l'Equipement, 1974. 
REVL-1976, núm. 190. IZQUIERDO DE BARTOLOME, RAFAEL. LOS PLANES DE CIRCULACION C...

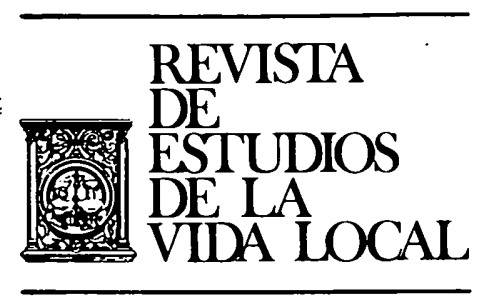

\section{CRONICAS}


REVL-1976, núm. 190. IZQUIERDO DE BARTOLOME, RAFAEL. LOS PLANES DE CIRCULACION C...

REVL-1976, núm. 190. IZQUIERDO DE BARTOLOME, RAFAEL. LOS PLANES DE CIRCULACION C... 